\title{
Aprendizaje tecnológico. Perspectiva de una unidad informática universitaria
}

JoSNel MARTÍNEZ GARCÉS ${ }^{1}$

RECIBIDO: 11/01/2017 ACEPTADO: 18/05/2017

\begin{abstract}
RESUMEN
El presente trabajo tiene por objetivo describir el aprendizaje tecnológico en la Unidad de Informática de la Universidad Rafael Urdaneta (Venezuela). Para lograrlo se realizó una investigación descriptiva, de campo y no experimental utilizando un cuestionario estructurado por diecisiete (17)ítems como instrumento de recolección de datos. La población estuvo integrada por todo el personal que labora en la referida unidad, el cual asciende a un total de tres (3) informantes clave. Los resultados permiten concluir que en la Unidad de Informática de la Universidad Rafael Urdaneta el aprendizaje tecnológico es un elemento que está presente en un grado medianamente alto como estrategia gerencial en su planificación organizacional, asimismo las actividades propias del aprendizaje tecnológico se desarrollan en un grado medianamente bajo pero, en consecuencia, se hace evidente como el aprendizaje tecnológico si se promueve y desarrolla entro los miembros que integran la unidad estudiada.
\end{abstract}

Palabras clave: aprendizaje tecnológico; actividades de aprendizaje; estrategia gerencial.

TECHNOLOGICAL LEARNING.

PERSPECTIVE OF A UNIVERSITY COMPUTER UNIT

\section{ABSTRACT}

The present paper aims to describe technological learning in the Computer Unit of the Rafael Urdaneta University (Venezuela). To achieve this, a descriptive, field and non-experimental investigation was carried out using a questionnaire structured by seventeen (17) ítems as a data collection instrument. The population was made up of all the personnel working in the referred unit, which amounted to a total of three (3) key informants. The results allow to conclude that in the Computer Unit of the Rafael Urdaneta University technological learning is an element that is present in a medium-high degree as a managerial strategy in its organizational planning, also the activities of technological learning are developed to a degree Moderate-low but, consequently, technological learning if it is promoted and developed among the members that integrate the unit studied.

Keywords: technological learning; learning activities; management strategy.

\section{INTRODUCCIÓN}

La era del conocimiento que estamos viviendo ha generado espacios propicios para la creación y desarrollo de tecnologías. Estas se han filtrado a todos los espacios donde el hombre habita como los hogares, empresas, industrias, gobiernos, entre otros, situación que ha generado la necesidad de aprender tecnología. Sin embargo, muchos autores consideran que las tecnologías no dependen de las ciencias, pues la datan con una antigüedad mayor a estas casi a la par con la génesis del hombre.

Bajo una óptica muy concreta se puede definir la tecnología como el conjunto de conocimientos que hacen posible la creación de productos y el diseño de los procesos que hacen esto posible (Cegarra, 2012). Esta exposición lleva implícita la necesidad de que el hombre tenga el conocimiento y las habilidades para saber-hacer y saber-usar la tecnología. En este sentido, el aprendizaje tecnológico juega un papel medular para lograrlo.

En este sentido, la presente investigación tiene por objetivo describir el aprendizaje tecnológico. Para lograrlo, se decidió escoger una unidad de análisis con características que permitieran comprobar si dentro de esta se llevan a cabo procesos propios del aprendizaje tecnológico. Por ello se decidió estudiar la Unidad de Informática de la Universidad Rafael Urdaneta (institución de educación superior privada y sin fines de lucro), la cual se encuentra ubicada en la ciudad de Maracaibo del estado Zulia (Venezuela); esta unidad está integrada por tres analistas de computación. Al momento del desarrollo del trabajo la unidad carecía de una estructura organizativa departamental definida, de manera que todos sus miembros ocupan un mismo rango jerárquico.

\section{METODOLOGÍA}

Para el desarrollo del trabajo se aplicó una metodología descriptiva con un diseño de campo, no experimental y transeccional. La población estuvo integrada por el total de personas que laboran en la Unidad de Informática de la Universidad Rafael

1 Licenciado en Contaduría Pública. Magister Scientiarum en Gerencia de Empresas, mención Gerencia Financiera. Magister Scientiarum en Planificación y Gerencia de Ciencia y Tecnología. Doctorando del programa Ciencias Sociales, mención Gerencia. Investigador adscrito al Centro de Estudios de la Empresa y al Centro Socioeconómico del Petróleo y Energías Alternativas de la Facultad de Ciencias Económicas y Sociales de la Universidad del Zulia (Venezuela). E-mail: josnel_martinez@ffces.luz.edu.ve 
Urdaneta (Venezuela), los cuales ascienden a tres (3) informantes clave; por esta razón, los datos que se mostrarán en el apartado de Resultados y Discusión han de ser considerados como las percepciones globales de todos los miembros que integran la referida Unidad. Asimismo, es importante señalar que dichos informantes clave poseen los conocimientos y las experiencias requeridas que los convierten en candidatos idóneos para abordar el objetivo de la presente investigación. En vista de que la población fue totalmente accesible no se aplicaron técnicas de muestreo. La observación de la variable se efectuó en el mes de marzo del año 2017.

Se recurrió a la encuesta como técnica de investigación y al cuestionario como herramienta para la recolección de datos. Este último estuvo integrado por 17 ítems, con opciones de respuesta de escala Likert, que permitieron medir la variable aprendizaje tecnológico dentro de la Unidad de Informática de la Universidad Rafael Urdaneta (Venezuela). En este sentido, la Tabla 1 muestra las alternativas de respuesta, su valor absoluto y su rango.

Tabla 1. Alternativas de respuesta y Rango para el instrumento de recolección de datos a través del cual se midió la variable Aprendizaje Tecnológico

\begin{tabular}{|l|c|c|}
\hline $\begin{array}{c}\text { Alternativa de } \\
\text { respuesta }\end{array}$ & Valor absoluto & Rango \\
\hline $\begin{array}{l}\text { Totalmente de } \\
\text { acuerdo }\end{array}$ & 4 & $3.26-4.00$ \\
\hline $\begin{array}{l}\text { Medianamente } \\
\text { de acuerdo }\end{array}$ & 3 & $2.51-3.25$ \\
\hline $\begin{array}{l}\text { Medianamente } \\
\text { en desacuerdo }\end{array}$ & 2 & $1.76-2.50$ \\
\hline $\begin{array}{l}\text { Totalmente en } \\
\text { desacuerdo }\end{array}$ & 1 & $1.00-1.75$ \\
\hline
\end{tabular}

Fuente: elaboración propia (2017).

Para el procesamiento de la información se utilizó la estadística descriptiva para agrupar los datos de acuerdo a su frecuencia absoluta y frecuencia relativa, de manera que posteriormente se hiciera más simple el proceso para el cálculo de la media aritmética que permitió definir las tendencias de las respuestas. Una vez hecho este proceso, se construyeron las tablas que recogen los resultados considerando las dos dimensiones del aprendizaje tecnológico: como estrategia gerencial y como capacidad empresarial.

\section{APRENDIZAJE TECNOLÓGICO}

Para Martínez y col. (2013), las capacidades tecnológicas -es decir, la acumulación, el uso y la transferencia de conocimientos para la creación de nuevos productos y procesos- junto a sus consecuentes ventajas competitivas son el resultado del aprendizaje tecnológico que adquieren las organizaciones en el marco de su interacción con otros agentes del contexto. Este proceso no solo se da en las personas, sino también en las organizaciones.

Bajo una perspectiva más amplia, González y Sánchez (2011) conceptualizan el aprendizaje tecnológico como aquel proceso colectivo donde se acumulan el conocimiento y la experiencia de las personas y las organizaciones, en el marco de la formación profesional dentro de la entidad, considerando al capital humano como un valor agregado en los procesos estratégicos de modernización tecnológica.

En este sentido, Carvajal (2011) considera que el aprendizaje tecnológico se fundamenta en cuatro pilares de la cultura tecnológica:

a) La creación de una conciencia sobre la presencia de la tecnología dentro de la sociedad como potenciador de actitudes positivas hacia ella.

b) La promoción del conocimiento y estudio de los posibles impactos de las tecnologías dentro de la sociedad.

c) La participación del usuario en la toma de decisiones sobre cuestiones tecnológicas, y no visto como un simple manipulador de estas.

d) La creación de ambientes idóneos para el desarrollo tecnológico.

Bajo este contexto, queda claro queel aprendizaje tecnológico se dará como un proceso rápido y eficaz siempre que esté enmarcado en la conversión del conocimiento explícito -aquel que se encuentra codificado y puede ser transmitido a través de lenguajes formales y sistemáticos- y tácito -originado en la mente del hombre y por ende difícilmente transmitido a no ser por medio de la acción y la participación en determinado contexto- (Garzón e Ibarra, 2013).

Ugas (2011) considera que el aprendizaje tecnológico permite hacer frente a los desafíos tecnológicos y de información ya que propicia el desarrollo 
de competencias y habilidades que incentivan la creación de conocimiento para emplearlo de manera eficiente y compartirlo con los miembros de la organización.

De esta manera se evidencia que el aprendizaje tecnológico es la acumulación de conocimientos y experiencias basados en la cultura tecnológica a través de los cuales se puede generar valor dentro de la organización con la finalidad de desarrollar competencias que permitan usar dichos conocimientos para la consecución de los objetivos estratégicos relacionados a la modernización tecnológica.

\section{RESULTADOS Y DISCUSIÓN}

Conti (2013) considera que las estrategias gerenciales integran el marco estratégico de la planificación organizacional, el cual puede descomponerse en cinco elementos clave: la misión, la visión, los objetivos, los valores y las políticas. La consideración del aprendizaje tecnológico dentro de estos componentes permite su desarrollo como parte de las metas finales de la organización.

a) Misión. A través de la misión se identifica la tarea básica que desarrolla una organización -o un componente de ella-, de manera que todos los establecimientos debidamente organizados deben poseer una misión (Koontz, 2003). De manera similar, Münch (2011) sostiene que la misión es una descripción de la razón de existencia de un negocio: lo que hace y a lo que se dedica. Para Serna (2000) la misión es lo que distingue a una organización de otra en cuanto a sus operaciones, productos o recurso humano. Por ello, Conti (2013) sostiene que una misión apropiadamente formulada debe evidenciar la tipología del negocio, la necesidad que satisface en la sociedad, su contribución al desarrollo económico, entre otros.

b) Visión.Es el futuro que se desea para la organización (Luna, 2010). La misión describe las aspiraciones para el futuro, define el curso de las estrategias y fija la dirección a largo plazo (Thompson y col., 2012). Francés (2001) añade que a través de ella se define el gran objetivo al cual dirigir los esfuerzos. En ella, de alguna u otra forman, convergen la misión y los objetivos de la organización los cuales se hacen tangibles cuando se convierten en metas específicas. Es el componente principal de la organización inteligente donde los empleados buscan una meta que alcanzar (Conti, 2013).

c) Objetivos.Son los fines que se persiguen mediante una determinada actividad dentro de la empresa. Es decir, la meta final que se persigue mediante la organización, dirección, control e integración del personal (Koontz, 2003). En este sentido, Luna (2010) considera que una vez la empresa conozca su misión y valores puede definir qué metas alcanzar en determinado período de tiempo, proporcionando así una dirección a las personas vinculadas a la organización. Por esta razón, Conti (2013) sostiene que los empleados están en la necesidad de definir estándares para evaluar su desempeño, lo que se logra a través de los objetivos, que pueden ser medidos mediante el margen de utilidad, la tasa de crecimiento, la participación en el mercado, el nivel de satisfacción de los clientes, el desarrollo e innovación de productos, entre otros.

d) Valores.Desde el punto de vista individual, para Stephen (1994) los valores representan una condición básica de conducta específica donde se define, de manera altamente subjetiva, lo que puede ser correcto, bueno o deseable. Estos están compuestos por atributos medidos a través de su contenido e intensidad. Por su parte, Conti (2013) considera que los valores son sinónimos de las actitudes generales que definen el comportamiento de la gente y las organizaciones, e integran la jerarquía del sistema de valores.

e) Políticas.Son enunciados o criterios que sirven de orientación para la toma de decisiones, aunque a veces no son tan formales sino que se desprenden de las acciones de los gerentes (Koontz, 2003). Por su parte, Münch (2011) considera que las políticas son lineamientos establecidos para poder lograr la sinergia de procesos necesaria que permita optimizar la toma de decisiones en función de lo que la organización desee alcanzar. Esta idea es respaldada por Conti (2013), quien afirma que establecer políticas bien definidas es importante porque sirven de herramientas para el logro de las metas y viabilizan la elaboración de planes concretos para el alcance de los objetivos.

En base a esto, a continuación la Tabla 2 presenta los resultados de medir el aprendizaje tecnológico como estrategia gerencial en la Unidad de Informática de la Universidad Rafael Urdaneta (Venezuela). Cabe destacar que los resultados que recoge la Tabla 2 representan las percepciones de los encuestados en relación a los componentes de la estrategia gerencial como potenciadores del aprendizaje tecnológico, y en ningún caso representan 
un cuestionamiento a si estos están adecuadamente organizados y/o alineados al objetivo estratégico de la organización como instrumentos o estándares que puedan articular la creación de conocimiento y su aplicación dentro de los procesos desarrollados.

Tabla 2. Aprendizaje tecnológico como estrategia gerencial en la unidad de informática de la Universidad Rafael Urdaneta

\begin{tabular}{|l|c|}
\hline \multicolumn{1}{|c|}{ Componentes } & Media aritmética \\
\hline Misión & 2.33 \\
\hline Visión & 2.67 \\
\hline Objetivos & 3.33 \\
\hline Valores & 3.00 \\
\hline Políticas & 3.33 \\
\hline \multicolumn{2}{|c|}{ Media de la dimensión } \\
\hline
\end{tabular}

Fuente: elaboración propia (2017).

Los resultados de la Tabla 1 muestran que de los elementos de la planificación organizacional de la Unidad de Informática de la Universidad Rafael Urdaneta, la misión arrojó una media aritmética de 2.33, demostrando que los entrevistados están medianamente en desacuerdo con que esta fomente el aprendizaje tecnológico. Sin embargo, la media aritmética del componente visión fue de 2.67 , de manera que están medianamente de acuerdo con que esta sí incluye el aprendizaje tecnológico como una meta que se quiere lograr en el futuro. Por otro lado, los objetivos, los valores y las políticas develaron una media aritmética de 3.33; 3.00 y 3.33, respectivamente, lo que permite inferir que los entrevistados están totalmente de acuerdo en que estos componentes promueven el aprendizaje tecnológico entre los empleados.

De esta manera, conjugando todos los elementos que integran la planificación organizacional, a través de una media aritmética de 2.93 se demuestra que los entrevistados están medianamente de acuerdo con que el aprendizaje tecnológico es una estrategia gerencial en la Unidad de Informática de la Universidad Rafael Urdaneta (Conti, 2013).

En otro orden de ideas, para Villavicencio y col. (1995), el aprendizaje tecnológico también puede ser una traducción de las capacidades empresariales. En este sentido, consideran que el mismo se puede lograr a través de seis actividades fundamentales: a) búsqueda de información especializada de opciones tecnológicas; b) negociación de tecnología; c) adaptación o modificación de equipos, partes y piezas; d) fabricación propia de equipos y partes; e) desarrollo de nuevos productos; y f) me- jora de los procesos o diseño de nuevos procesos productivos.

a) Búsqueda de información especializada de opciones tecnológicas. Representa para la organización un aviso de los posibles oferentes de tecnología (Villavicencio y col., 1995). Esta debe ser una actividad permanente, incluso las empresas deberían considerar la posibilidad de crear una unidad de gestión para buscar, analizar, almacenar y difundir información basada en sus requerimientos tecnológicos (Ávalos, 1992). En este sentido, dicha búsqueda debe considerar fuentes nacionales e internacionales que resulten novedosas y oportunas; estas pueden estar representadas por documentación impresa, electrónica o a través de relaciones inter-empresariales (González y Sánchez, 2011).

b) Negociación de tecnología. Aspecto fundamental para definir las ventajas y beneficios que se puedan obtener tras adquirir determinada tecnología (Villavicencio y col., 1995). González y Sánchez (2011) sostienen que esta actividad se asocia con la contratación de productos y servicios relacionados estrictamente a soluciones tecnológicas. En este sentido, Martínez y col. (2013) consideran que la frecuencia y la calidad de las relaciones proveedor-empresa-cliente propician la construcción de círculos virtuosos de innovación y mejora. Para Ávalos (1992), esta negociación debe apuntar al aprovechamiento máximo de la tecnología presente en la organización de manera que se puedan optimizar los procesos productivos.

c) Adaptación o modificación de equipos, partes y piezas. Para Villavicencio y col. (1995), es una actividad casi particular de las empresas que funcionan en países cuasi-industrializados,desarrollada de manera empírica y con una escasa o nula documentación y sistematización de ella. Martínez y col. (2013) añaden que esta actividad permite a las organizaciones elevar sus niveles de productividad y reducir sus niveles de costos. Por su parte, González y Sánchez (2011) consideran que esta tarea no obliga a la obtención de un producto tangible, sino que se orienta a los conocimientos asociados a una tecnología interna específica, ello podría implicar contratación de asesores, diseño de manuales, creación de bases de datos, entre otros.

d) Fabricación propia de equipos y partes. Se suele derivar de la adaptación o modificación 
de equipos, partes y piezas para resolver las deficiencias de equipos y repuestos (Villavicencio y col., 1995). En otras palabras, es la producción por medios propios de aquellos equipos que normalmente se suelen adquirir de terceros, aunque no siempre se trata de tecnología dura sino que en algunos casos puede ser para desarrollar tecnologías blandas (González y Sánchez, 2011). En este sentido, Martínez y col. (2013) afirman que si se usan de manera eficiente, las tecnologías pueden llevar a los actores sociales a diseñar propuestas para su mejora.

e) Desarrollo de nuevos productos. Villavicencio y col. (1995) consideran que esto se refiere a las modificaciones menores que se le puedan realizar a determinado producto, pero que resulta una novedad para la organización o el mercado así como al desarrollo de un producto estrictamente nuevo. González y Sánchez (2011) sostienen que a través del desarrollo de nuevos productos se puede potenciar la generación de conocimientos y la satisfacción de requerimientos empresariales sin la necesidad de contar con un ambiente productivo especializado. Para Martínez y col. (2013) el aprendizaje es un elemento clave para la creación de nuevas propuestas que impulsen la competitividad.

f) Mejora de los procesos o diseño de nuevos procesos productivos. Para González y Sánchez (2011) es la capacidad de innovar a gran escala en los procesos organizacionales. Estudiar el proceso productivo permite a los miembros de la empresa poseer un conocimiento pleno de sus detalles para poder detectar con facilidad sus puntos de mejoría. Po esta razón, Villavicencio y col. (1995) consideran que es la actividad más compleja dentro de la organización.

Por otro lado, Ugas (2011) considera que muchas veces la tecnología en sí se convierte en una barrera que dificulta su aprendizaje. Entre los principales factores se destacan: la complejidad tecnológica, la diversidad tecnológica, la interdependencia de tecnologías, las tecnologías propietarias, la incompatibilidad tecnológica y la obsolescencia tecnológica.

Tomando esto en consideración, a continuación la Tabla 3 presenta los resultados de medir el aprendizaje tecnológico como capacidad empresarial en la Unidad de Informática de la Universidad Rafael Urdaneta (Venezuela).
Tabla 3. Aprendizaje tecnológico como capacidad empresarial en la unidad de informática de la Universidad Rafael Urdaneta

\begin{tabular}{|c|c|}
\hline Indicador & $\begin{array}{l}\text { Media arit- } \\
\text { mética }\end{array}$ \\
\hline $\begin{array}{l}\text { Búsqueda de información especiali- } \\
\text { zada de opciones tecnológicas }\end{array}$ & 2.33 \\
\hline Negociación de tecnología & 2.33 \\
\hline $\begin{array}{l}\text { Adaptación o modificación de equi- } \\
\text { pos, partes y piezas }\end{array}$ & 2.00 \\
\hline Fabricación propia de equipos y partes & 2.00 \\
\hline Desarrollo de nuevos productos & 3.33 \\
\hline $\begin{array}{l}\text { Mejora de los procesos o diseño de } \\
\text { nuevos procesos productivos }\end{array}$ & 3.33 \\
\hline Media de la dimensión & 2.50 \\
\hline
\end{tabular}

Fuente: elaboración propia (2017).

Los resultados de la Tabla 3 muestran que de las actividades desarrolladas dentro de la Unidad de Informática de la Universidad Rafael Urdaneta, quienes laboran ahí están medianamente en desacuerdo que les ha sido posible la búsqueda de información especializada de opciones tecnológicas (media aritmética de 2.33); la negociación de tecnología (media aritmética de 2.33); la adaptación o modificación de equipos, partes y piezas (media aritmética de 2.00); y la fabricación propia de equipos y partes (media aritmética de 2.00). Por otro lado, a través de una media aritmética de 3.33 se puede notar cómo están totalmente de acuerdo en que sí han podido desarrollar nuevos productos; mejorar los procesos; y diseñar nuevos procesos productivos.

En una convergencia de todas las actividades relacionadas al aprendizaje tecnológico se puede concluir,a través de una media aritmética de 2.50, que los entrevistados están medianamente en desacuerdo con que este sea un reflejo de las capacidades empresariales de la Unidad de Informática de la Universidad Rafael Urdaneta (Villavicencio y col., 1995).

Adicionalmente, se pudo conocer que un $100 \%$ de los encuestados considera la diversidad tecnológica como un factor limitante del aprendizaje tecnológico. Por otro lado, a menor grado un $66,67 \%$ considera que otros factores limitantes adicionales han sido la complejidad tecnológica, la interdependencia de tecnologías y la incompatibilidad tecnológica. Como expone Ugas (2011), estos aspectos son elementos que dificultan el aprendizaje tecnológico. 


\section{CONCLUSIONES}

A través de los resultados expuestos se puede concluir que:

- El aprendizaje tecnológico es una estrategia gerencial que está presente en un nivel medianamente alto dentro de todos los componentes de la planificación empresarial dentro de la Unidad de Informática de la Universidad Rafael Urdaneta. En primer lugar a través de los objetivos, valores y políticas establecidos, seguidos por la visión y a menor grado dentro de la misión.

- El aprendizaje tecnológico como capacidad empresarial se considera medianamente bajo, permitiendo desarrollar sólo unas pocas actividades que lo potencian, destacándose entre ellas el desarrollo de nuevos productos, la mejora de procesos y el diseño de nuevos procesos productivos

- Hay factores que han limitado su aprendizaje tecnológico, y el principal de ellos ha sido la diversidad tecnológica

- En síntesis, el aprendizaje tecnológico puede describirse como medianamente desarrollado en la Unidad de Informática de la Universidad Rafael Urdaneta.

\section{REFERENCIAS BIBLIOGRÁFICAS}

[1] Ávalos, I. (1992). Aproximación a la gerencia de la tecnología en la empresa. Caracas, Venezuela: Instituto de Estudios Superiores en Administración.

[2] Carvajal, Á. (2011). La cultura tecnológica como base de las capacidades y el aprendizaje tecnológico. Revista Humanidades, 1, 1-13.

[3] Cegarra, J (2012). La tecnología. España: Ediciones Díaz de Santos.

[4] Conti, G. (2013). Aprendizaje tecnológico: aspectos estratégicos del aprendizaje tecnológico presente en estudios integrados de yacimientos en empresas mixtas del sector petrolero. Revista del Centro de Investigación de Ciencias Administrativas y Gerenciales, 11(1), 74.83 .
[5] Francés, A. (2006). Estrategia y Planes para la Empresa con el Cuadro de Mando Integral. Ciudad de México, México:Editorial Prentice Hall.

[6] Garzón, M. e Ibarra, A. (2013). El aprendizaje tecnológico como acelerador de la innovación. Escenarios, 11(1),57-77.

[7] González, R. y Sánchez, J. (2011). Aprendizaje tecnológico: las actividades del aprendizaje tecnológico en las gerencias de informática de las empresas mixtas del sector petrolero del estado Zulia. Revista Venezolana de Ciencia y Tecnología 1(2), 2-12.

[8] Koontz, H. y Weihrich, H. (2004). Administración: una perspectiva global.México: Editorial McGrawHill.

[9] Luna, A. (2010). Administración estratégica. Ciudad de México, México: Grupo Editorial Patria.

[10] Martínez, A.; García, A. y Santos, G. (2013). Aprendizaje tecnológico en la industria manufacturera de Guanajuato. Frontera Norte, 25(50), 187-212.

[11] Münch, L. (2011). Planeación estratégica: El rumbo hacia el éxito.Ciudad de México, México: Editorial Trillas.

[12] Serna, H. (2000). Planeación y gestión estratégica. Bogotá, Colombia: Editorial RAM.

13] Stephen, J. (1994). Cómo valorar la conducta especifica. Buenos Aires, Argentina: Editorial Centro Americano.

[14] Thompson, A.; Strickland III, A. y Gamble, J.(2012) Administración Estratégica. Textos y Casos. Ciudad de México, México: Editorial McGrawHill.

[15] Ugas, Luis (2011). Aprendizaje tecnológico e informacional para desarrollar una cultura de enseñanza en organizaciones inteligentes. Revista del Centro de Investigación de Ciencias Administrativas y Gerenciales, 9(1), 6289.

[16] Villavicencio, D.; Arvanitis, R. y Minsberg, L. (1995). Aprendizaje tecnológico en la industria química mexicana. Perfiles Latinoamericanos, 7, 121-148. 